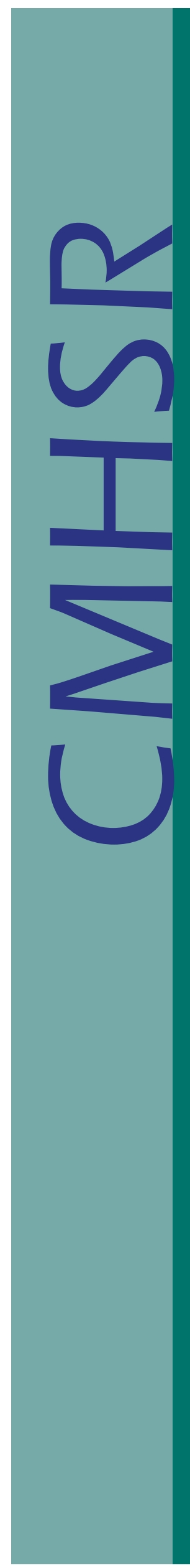

\title{
Drug-Related Arrests among Persons with Serious Mental IIIness
}

William H. Fisher, Ph.D., Kristen Roy-Bujnowski, MA, Albert J. Grudzinskas, Jr., JD., Jonathan C. Clayfield, MA, LMHC, \& Steven Banks, Ph.D.

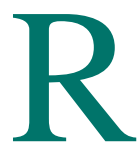

ecent epidemiological data reveal an extremely high prevalence of co-occurring substance abuse among persons with serious psychiatric disorders. ${ }^{1}$ Problems associated with co-occurring substance abuse and mental illness have been a long-standing focus of the psychiatric and mental health services research literature. In this literature, abuse of alcohol and drugs is often treated as a single problem. Clearly, both substances can cause persons with serious mental illness to lapse from treatment, increase their risk of involvement with violence, and exacerbate risk of homelessness. ${ }^{2}$ However, the consequences of illicit drug use are arguably greater. Sharing needles, for example, can greatly elevate risk of contracting HIV and other infectious diseases, and can promote involvement in other illegal activities and social networks that reinforce antisocial behaviors.

An additional, less frequently discussed aspect of drug use is its illegality. Unlike alcohol, which is legal when used by adults, simple possession of illicit drugs, even in small quantities, is illegal. Indeed, simply being where drugs are known to be present is grounds for arrest in some jurisdictions, and convictions on charges beyond simple possession can result in some cases in draconian prison sentences as well as additional post-incarceration penalties. Among these penalties is exclusion from Section Eight housing, ${ }^{3}$ an important source of residential support for persons with serious mental illness. Despite the seriousness of these consequences, there has been little discussion of the criminal justice aspects of drug use among persons with serious mental illness. This brief attempts to provide a starting point for such a discussion.

๑) 2007 Center for Mental Health Services Research Department of Psychiatry

University of Massachusetts Medical School

\section{Method}

We examined data on a cohort of individuals over the age of $18(\mathrm{~N}=13,816)$ who received adult services (either inpatient, residential or case management services) from the Massachusetts Department of Mental Health in 1991. Cohort members were eligible for these services based on their having a diagnosis of serious and persistent mental illness, a history of functional disability and multiple psychiatric hospitalizations. The Massachusetts Criminal Offender Record Information system provided arrest data for cohort members spanning just less than 10 years following the cohort's identification. (Additional details have been published elsewhere) ${ }^{4}$

\section{Results}

Some 720 individuals, or $5.2 \%$ of the cohort, were arrested at least once on a drug-related charge over the 10-year observation period, accumulating a total of 9,357 charges. Closer inspection of the age distribution of these arrests indicated, not surprisingly, perhaps, that drug charges are most highly concentrated among persons under the age of 40 , a group with a $30 \%$ drug arrest rate over 10 years. Table 1 , shown on the next page of this brief, summarizes the charges associated with drug arrests for the entire cohort. The majority of charges are for simple possession or being present where drugs were kept. At the same time, roughly $20 \%$ of charges reflected involvement with drug distribution or manufacturing.

\section{Discussion}

Several factors must be considered in viewing these data. Arrests are neither findings of guilt nor convictions, nor do they represent the "true" prevalence of drugrelated behaviors. Also, this cohort included persons whose psychiatric illnesses were severe and persistently disabling enough to meet the state's stringent eligibility criteria for receiving services. This point should be kept in mind when considering the generalizability of these data to the broader population of persons with serious psychiatric disorders. 
Drug - related charges $(\mathrm{N}=9,357)$ filed against 720 cohort members, by type of activity and drug class

\begin{tabular}{|c|c|c|}
\hline & $\mathrm{N}$ & Pct of Charges \\
\hline & \multicolumn{2}{|c|}{$\begin{array}{c}\text { Possession/Knowingly being present where } \\
\text { drugs are kept }\end{array}$} \\
\hline \multicolumn{3}{|l|}{ Substance } \\
\hline Class A codeine, heroin and morphine, etc. & 998 & 10.6 \\
\hline Class B opium and cocaine & 2259 & 24.1 \\
\hline Class $\mathrm{C}$ barbiturates, as well as hallucinogens & 135 & 1.4 \\
\hline Class D phenobarbital and marijuana, & 2319 & 24.8 \\
\hline Class E non-narcotic medicinal drugs & 225 & 2.4 \\
\hline Counterfeit Drugs & 9 & .1 \\
\hline \multirow[t]{2}{*}{ Other } & 1363 & 14.5 \\
\hline & \multicolumn{2}{|c|}{$\begin{array}{c}\text { Possession with intent to distribute/ } \\
\text { Trafficking }\end{array}$} \\
\hline \multicolumn{3}{|l|}{ Substance } \\
\hline Class A codeine, heroin and morphine, etc. & 338 & 3.6 \\
\hline Class B opium and cocaine & 632 & 6.7 \\
\hline \multicolumn{3}{|l|}{ Other } \\
\hline Within 1000 feet of a school & 718 & 7.6 \\
\hline \multicolumn{3}{|l|}{ Manufacturing } \\
\hline Class A through $\mathrm{E}$ & 291 & 3.8 \\
\hline
\end{tabular}

*Massachusetts General Laws (Chapter 94C: Section 31) recognizes 5 "classes" (A through E) of illegal substances, possession and/or distribution of which carry different levels of penalty.

The data presented here argue for adding risk of significant criminal justice involvement to previously identified problems associated with substance use among persons with severe mental illness. Some charges observed here involve possession of marijuana or other "recreational" drugs and may only carry minor penalties, especially if they are first offenses. Other charges involving "harder" drugs or manufacturing and/or "possession with intent to distribute" carry much harsher penalties. It is unlikely that these arrestees are "major drug dealers"; it is more likely that they work as processors or as "mules," transporting drugs on behalf of dealers in exchange for drugs or cash. Some accounts suggest these activities hold multiple attractions. Whether our cohort members' activities pay off in drugs, money, social status or all three cannot be discerned here. ${ }^{5}$

Our data lack the detail required to make definitive statements about the nature and etiology of drug-related behaviors. We would hope, however, that these findings would stimulate further research in this area. From the perspective of the mental health system, it would be useful to understand how individuals with severe mental illness become involved in drug-related activities, particularly those that have serious criminal justice consequences. If it is the economic or social attractiveness of such involvement that lures these individuals into this kind of activity, social skills training protocols might be developed that would help in recognizing these attractions and make clear the risks associated with them. Doing so requires that we formulate a research agenda that will foster that understanding, taking into account the demographic and other features of those at greatest risk and tailoring clinical and social intervention and prevention strategies.

\section{References}

1. Kessler, R. C., McGonagle, K. A., Zhao, et al. (1994) "Lifetime and 12-month prevalence of DSM-IIIR psychiatric disorders in the United States: Results from the National Comorbidity Survey.” Archives of General Psychiatry, 51, 8-19.

2. RachBeisel, J., Scott, J., \& Dixon, L. (1999). “Co-occurring severe mental illness and substance use disorders: A review of recent Research.” Psychiatric Services, 50, 1427-1434.

3. Department of Housing and Urban Development (2001). Screening and Eviction for Drug Abuse and Other Criminal Activity; Final Rule; 24 CFR Parts 5 et al. Federal Register, May 24, 2001. Washington, DC: National Archives and Records Adminstration.

4. Fisher, W. H,, Roy-Bujnowski, K., Grudzinskas, A. J., Clayfield, C., Banks, S. M., \& Wolff, N. (2004). "Patterns and prevalence of arrest in a statewide cohort of mental health care consumers." Psychiatric Services, 57, 1623-1628.

5. LeBlanc, A. N. (2003). "Random family: Love, drugs, trouble and coming of age in the Bronx." New York: Scribner.

Visit us on-line at www.umassmed.edu/cmhsr 